FROM: Luijt DS, Schirm J, Savelkoul PH, Hoekstra A. Risk of infection by reprocessed and resterilized viruscontaminated catheters: an in-vitro study. Eur Heart $J$ 2001;22:378-384.

\section{Ethylene Oxide Exposure Determinants}

LaMontagne and Kelsey, from the Dana-Farber Cancer Institute, Boston, conducted a study to identify determinants of workplace exposures to ethylene oxide to assess the effect OSHA's 1984 ethylene oxide standard. An in-depth survey of all hospitals in Massachusetts that used ethylene oxide from 1990 through 1992 (96\% participation, $\mathrm{n}=90$ ) was conducted. Three types of exposure events were modeled with logistic regression: exceeding the 8-hour action level, exceeding the 15-minute excursion limit, and worker exposures during unmeasured accidental releases. Covariates were drawn from data representing an ecologic framework including direct and indirect potential exposure determinants.

After adjustment for frequencies of ethylene oxide use and exposure monitoring, a significant inverse relation was observed between exceeding the action level and the use of combined sterilizer-aerators, an engineering control technology developed after the passage of the OSHA standard. Conversely, the use of positive-pressure sterilizers employing ethylene oxide gas mixtures was strongly related to both exceeding the excursion limit and the occurrence of accidental releases.

These findings provide evidence of a positive effect of OSHA's ethylene oxide standard and specific targets for future prevention and control efforts.

FROM: LaMontagne AD, Kelsey KT. Evaluating OSHA's ethylene oxide standard: exposure determinants in Massachusetts hospitals. Am J Public Health 2001;91: 412-417.

\section{Hand Washing, Gowns, and Gloves Reduce Pediatric Transplant Infections}

Nosocomial infection is an important contributor to morbidity and mortality in pediatric solid-organ transplantation. The relative effect of protective gown and glove isolation was compared with strict hand washing in pediatric intensive care unit (PICU) patients with solid-organ transplantation in a prospective randomized study.

Children in a 23-bed PICU with solid-organ transplantation were enrolled into a gown and glove protective isolation protocol or a strict handwashing protocol. Children with current infection or known exposure to varicella were excluded from the study initially or at readmission.

The infection rate in the overall PICU population did not change significantly from the year before the study compared to during the study (2.1/100 vs $1.95 / 100$ patientdays; $P=.4$ ) The infection rate in the gown-and-glove group (2.3/100 patient-days) was reduced significantly compared with the pre-study infection rate in the transplant popula- tion (4.9/100 patient-days; $P=.0008)$. Strict hand washing also significantly reduced the infection rate in the transplant population $(3.0 / 100$ patient-days; $P=.008)$. Compliance with gowning and gloving was $82 \%$ and compliance with hand washing was $76 \%$ (compared to $22 \%$ before study $[P<.0001]$ and $52 \%$ after the study $[P<.0001])$. Despite an increased mean length of stay in the PICU in the gown-and-glove group $(P=.014)$, there was a trend toward reduction in the incidence of infection in the gown-andglove group.

The authors concluded that increased compliance with hand washing was associated with a reduction in nosocomial infections, and gown-and-glove isolation appeared to have an additional protective effect. Thus, some nosocomial infections may be preventable in the pediatric solid-organ transplantation population.

FROM: Slota M, Green M, Farley A, Janosky J, Careillo J. The role of gown and glove isolation and strict handwashing in the reduction of nosocomial infection in children with solid organ transplantation. Crit Care Med 2001;29:405-412.

\section{Computerized Detection of Infections}

Rocha and colleagues, from the University of Utah, Salt Lake City, conducted a study to analyze whether computer-generated reminders about infections could influence clinicians' practice patterns and consequently improve the detection and management of nosocomial infections. The conclusions produced by an expert system developed to detect and manage infections were presented to the attending clinicians in a pediatric hospital to determine whether this information could improve detection and management. Clinician interventions were compared before and after the implementation of the system. The responses of the clinicians (staff physicians, physician assistants, and nurse practitioners) to the reminders were determined by review of paper medical charts. Main outcome measures were the number of suggestions to treat and manage infections that were followed before and after the implementation of COMPISS (Computerized Pediatric Infection Surveillance System). The clinicians' opinions about the system were assessed by means of a paper questionnaire distributed following the experiment.

The results failed to show a statistical difference between the clinicians' treatment strategies before and after implementation of the system $(P>33$ for clinicians working in the emergency room and $P .45$ for clinicians working in the pediatric intensive care unit). The questionnaire results showed that the respondents appreciated the information presented by the system.

The authors concluded that the computer-generated reminders about infections were unable to influence the practice patterns of clinicians, citing methodological problems as a possible contributing factor.

FROM: Rocha BH, Christenson JC, Evans RS, Gardner RM. Clinicians' response to computerized detection of infections. J Am Med Inform Assoc 2001;8:117-125. 